\title{
COVID-19: changing patterns among neurosurgical patients from North India, efficacy of repeat testing, and inpatient prevalence
}

\author{
*Sushanta K. Sahoo, MCh, ${ }^{1}$ Sivashanmugam Dhandapani, MCh, ${ }^{1}$ Apinderpreet Singh, MCh,1 \\ Chandrashekhar Gendle, MCh, ${ }^{1}$ Madhivanan Karthigeyan, MCh, ${ }^{1}$ Pravin Salunke, MCh, ${ }^{1}$ \\ Ashish Aggarwal, MCh, ${ }^{1}$ Navneet Singla, MCh, ${ }^{1}$ Raghav Singla, MCh, ${ }^{1}$ Manjul Tripathi, MCh, ${ }^{1}$ \\ Rajesh Chhabra, MCh, ${ }^{1}$ Sandeep Mohindra, MCh, ${ }^{1}$ Manoj Kumar Tewari, MCh, ${ }^{1}$ \\ Manju Mohanty, PhD, ${ }^{1}$ Hemant Bhagat, DM, ${ }^{2}$ Arunaloke Chakrabarti, MD, ${ }^{3}$ and \\ Sunil Kumar Gupta, $\mathrm{MCh}^{1}$
}

Departments of ${ }^{1}$ Neurosurgery, ${ }^{2}$ Anesthesia, and ${ }^{3}$ Microbiology, Postgraduate Institute of Medical Education and Research (PGIMER), Chandigarh, India

OBJECTIVE COVID-19 has affected surgical practice globally. Treating neurosurgical patients with the restrictions imposed by the pandemic is challenging in institutions with shared patient areas. The present study was performed to assess the changing patterns of neurosurgical cases, the efficacy of repeated testing before surgery, and the prevalence of COVID-19 in asymptomatic neurosurgical inpatients.

METHODS Cases of non-trauma-related neurosurgical patients treated at the Postgraduate Institute of Medical Education and Research (PGIMER) before and during the COVID-19 pandemic were reviewed. During the pandemic, all patients underwent a nasopharyngeal swab reverse transcription-polymerase chain reaction test to detect COVID-19 at admission. Patients who needed immediate intervention were surgically treated following a single COVID-19 test, while stable patients who initially tested negative for COVID-19 were subjected to repeated testing at least 5 days after the first test and within 48 hours prior to the planned surgery. The COVID-19 positivity rate was compared with the local period prevalence. The number of patients who tested positive at the second test, following a negative first test, was used to determine the probable number of people who could have become infected during the surgical procedure without second testing.

RESULTS Of the total 1769 non-trauma-related neurosurgical patients included in this study, a mean of 337.2 patients underwent surgery per month before COVID-19, while a mean of 184.2 patients ( $54.6 \%$ of pre-COVID-19 capacity) underwent surgery per month during the pandemic period, when COVID-19 cases were on the rise in India. There was a significant increase in the proportion of patients undergoing surgery for a ruptured aneurysm, stroke, hydrocephalus, and cerebellar tumors, while the number of patients seeking surgery for chronic benign diseases declined. At the first COVID-19 test, 4 patients $(0.48 \%)$ tested were found to have the disease, a proportion 3.7 times greater than that found in the local community. An additional 5 patients tested positive at the time of the second COVID-19 test, resulting in an overall inpatient period prevalence of $1 \%$, in contrast to a $0.2 \%$ national cumulative caseload. It is possible that COVID-19 was prevented in approximately 67.4 people every month by using double testing.

CONCLUSIONS COVID-19 has changed the pattern of neurosurgical procedures, with acute cases dominating the practice. Despite the fact that the pandemic has not yet reached its peak in India, COVID-19 has been detected 3.7 times more often in asymptomatic neurosurgical inpatients than in the local community, even with single testing. Double testing displays an incremental value by disclosing COVID-19 overall in 1 in 100 inpatients and thus averting its spread through neurosurgical services.

https://thejns.org/doi/abs/10.3171/2020.9.FOCUS20705

KEYWORDS neurosurgery; COVID-19; RT-PCR; reverse transcription-polymerase chain reaction; repeat; isolation; quarantine

ABBREVIATIONS AVM = arteriovenous malformation; $C T$ = computed tomography; ICU = intensive care unit; PGIMER = Postgraduate Institute of Medical Education and Research; $P P E=$ personal protective equipment; $R T-P C R=$ reverse transcription-polymerase chain reaction; VP = ventriculoperitoneal.

SUBMITTED August 1, 2020. ACCEPTED September 18, 2020.

INCLUDE WHEN CITING DOI: 10.3171/2020.9.FOCUS20705.

* S.K.S. and S.D. share first authorship of this work. 
$\mathrm{C}$ OVID-19 has caused a paradigm shift in the medical and surgical care of patients. Governments in many countries have enforced lockdowns to restrict the spread of infection. Still, the number of COVID-19 cases is on the rise in many countries, including India. ${ }^{1}$

Medical facilities have been diverted toward managing the unprecedented situation brought about by the COVID-19 pandemic. Almost all hospitals worldwide were forced to suspend their elective surgeries and formulate new guidelines for patient admission, taking into consideration the level of the emergency, to prepare hospital areas for treating COVID-19 patients, protect their healthcare providers, and prevent crowding and the facilitated spread of COVID-19. ${ }^{2-4}$ This is even more relevant in institutions in developing countries, where many patients are made to share a patient area. Due to the dynamic nature of the spread of COVID-19, hospitals are continually updating protocols for case management. ${ }^{2,3}$

Despite scattered reports on the impact of COVID-19 in neurosurgery, the efficacy of COVID-19 testing protocols and the inpatient prevalence of COVID-19 in neurosurgical patients have not been studied in detail. This study was performed to evaluate the impact of COVID-19 on the number and types of non-trauma-related neurosurgical patients who are being treated at our institution to test the efficacy of repeated COVID-19 testing and to determine the comparative prevalence of COVID-19 among inpatients compared with that in the local community.

\section{Methods}

Ours is one of the largest neurosurgical tertiary care institutions catering to the needs of northern India, managing an average of 115 inpatient cases at a time. In India, the national lockdown was enforced by the government on March 23, 2020, to curb the spread of COVID-19. Our institution, the Postgraduate Institute of Medical Education and Research (PGIMER), was declared the COVID-19 center for the region, and a separate building inside the institution's complex was designated for it.

\section{Management Protocol}

In the pre-COVID-19 period, neurosurgery patients were admitted through outpatient clinics and from emergency departments. All patients were thoroughly evaluated clinically, underwent neuroimaging along with routine investigations, and were surgically treated. During the COVID-19 period (Fig. 1), all patients were admitted only through the emergency wing of neurosurgery. Before entering the emergency area, all patients and their relatives were asked to use protective measures, such as face masks and hand sanitizers, and advised to follow social distancing. They also underwent thermal screening followed by an assessment of the history of fever, respiratory complaints, travel to and from containment zones, and contact with COVID-19 patients in the last 14 days; highrisk patients were referred to the COVID-19 center within our institution.

Other patients who needed neurosurgical intervention or inpatient care were admitted to the "suspect area" of the emergency neurosurgery wing. Here patients were evalu- ated by neurosurgery team members who used protective measures such as N95 respirators, face shields, hand gloves, and impervious gowns. All patients were subjected to a nasopharyngeal swab reverse transcription-polymerase chain reaction (RT-PCR) test for SARS-CoV-2., They were then evaluated clinically, and the severity of their symptoms was documented while avoiding close physical examinations (e.g., the gag reflex). In addition to nasopharyngeal swabbing, the use of chest computed tomography (CT) has also been reported to diagnose symptomatic cases of COVID-19. However, considering the absence of respiratory symptoms, high caseloads, and resource constraints, a routine chest CT scan is not feasible for all neurosurgical patients admitted to our center. We primarily rely on the nasopharyngeal swab RT-PCR test to detect the presence of SARS-CoV-2.

Patients who needed immediate intervention underwent surgery following a single COVID-19 test. In contrast, after receiving a negative test, stable patients were transferred to the preoperative general ward, which is shared by other patients, where they were subjected to a second nasopharyngeal swab RT-PCR test at least 5 days after the first test and within 48 hours before the planned surgery. ${ }^{8}$ Neurosurgical procedures were carried out with all staff wearing FFP1 respirators, gloves, and gowns. ${ }^{9}$ As all patients underwent surgery after testing negative for COVID-19, only FFP1 respirators were used (due to limited availability, N95 respirators were reserved for managing suspected or COVID-19-positive cases). ${ }^{9}$ After surgery, all patients were transferred to the postoperative ward, where they shared cubicles with a restricted number of caregivers. They were all strictly observed for the development of any symptoms related to COVID-19.

Any patient designated positive by the first or second test was transferred to the COVID-19 center for further case management. At the same time, that particular area was sanitized and contained for 5 days, and staff members underwent risk assessment and were quarantined in case of close or significant exposure. At the end of 5 days, all patients in that area were further subjected to a nasopharyngeal swab RT-PCR test to detect SARS-CoV-2. All healthcare workers who came in contact with COVID-19positive patients were observed strictly for the development of symptoms related to COVID-19 over the next 14 days.

If the neurosurgery patient in the COVID-19 center was stable, surgery was rescheduled 4 weeks into the future pending two consecutive negative tests. Patients who needed urgent intervention underwent surgery in the dedicated COVID-19 operating room with all personnel wearing full personal protective equipment (PPE), including N95 respirators and face shields.

\section{Evaluation Methodology}

All non-trauma-related patients admitted to the Department of Neurosurgery between April 1, 2020, and August 15, 2020 (COVID-19 period), were evaluated and compared with similar patients who had been treated between January 1, 2020, and March 22, 2020 (preCOVID-19 period; historical controls). Patients treated between March 23 and March 31 were excluded, as most 


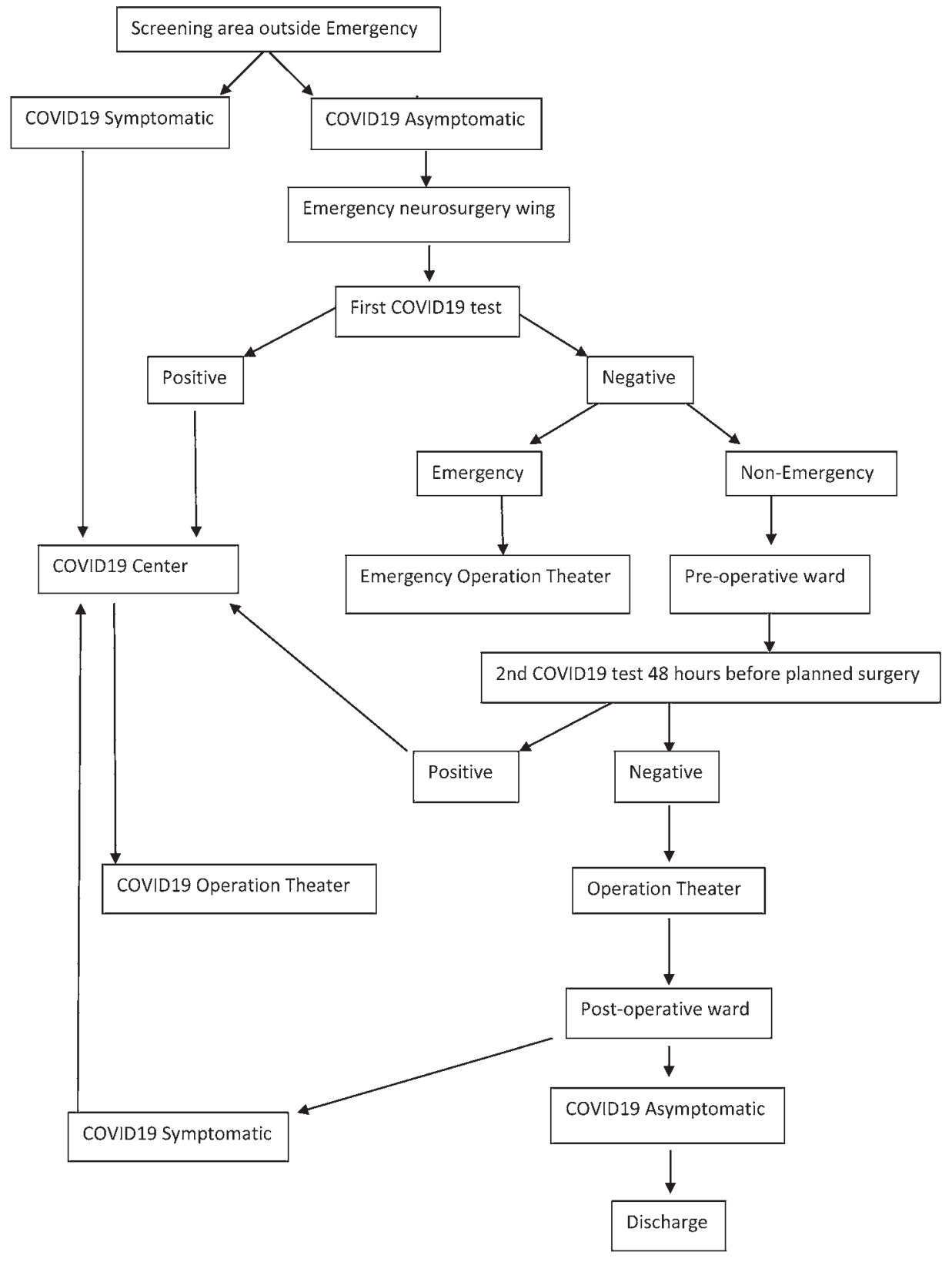

FIG. 1. Flowchart showing neurosurgery patients during the COVID-19 period.

of them were admitted before the COVID-19 restrictions. Patients were grouped according to pre-COVID-19 or COVID-19 period and compared for age, sex, and clinicoradiological diagnosis. The two groups were compared for statistically significant differences by using SPSS version 21 (IBM Corp.) with the chi-square test. Two-sided significance tests were used throughout, and the significance level was deemed $\mathrm{p}<0.05$.

The COVID-19 positivity rate was assessed among inpatients by using the first test and also the double testing strategy. The positivity rates were compared with the local period prevalence among regions covered by our center as well as with rates of national cumulative positivity.

The additional number of patients who were found to be COVID-19 positive by using the second test, after a negative first COVID-19 test, was used to determine the probable number of people who could have become infected by the surgical procedure without the second testing. This calculation considered that approximately 8 persons would come in close contact with the patient during surgery, who in turn could infect a number of people equal to the "effective reproduction number R0" over a mean incubation period of 7 days. ${ }^{10,11}$

\section{Results}

\section{Patient Populations}

A total of 1769 non-trauma-related neurosurgery pa- 
TABLE 1. Comparison of non-trauma-related neurosurgical patients surgically treated during pre-COVID-19 and COVID-19 periods

\begin{tabular}{lccc} 
& \multicolumn{2}{c}{ No. of Patients (\%) } & \\
\cline { 2 - 3 } \multicolumn{1}{c}{ Diagnostic Category } & Pre-COVID-19 $(83$ days $)$ & COVID-19 (137 days) & Changes in COVID-19 Period \\
\hline All categories & 933 & 829 & Decreased \\
\hline Glioma & $158(16.9)$ & $108(13.0)$ & Increased \\
\hline Ruptured aneurysms/AVM & $99(10.6)$ & $149(18.0)$ & Increased \\
\hline Cerebellar tumors & $21(2.3)$ & $33(4.0)$ & Increased \\
\hline Hydrocephalus & $221(23.7)$ & $263(31.7)$ & Decreased \\
\hline Cerebral metastasis & $57(6.1)$ & $14(1.7)$ & Decreased \\
\hline Calvarial meningiomas & $39(4.2)$ & $27(3.3)$ & Increased \\
\hline Stroke & $36(3.9)$ & $53(6.4)$ & Decreased \\
\hline Sellar-suprasellar tumors & $52(5.6)$ & $21(2.5)$ & Decreased \\
\hline Spinal disorders & $98(10.5)$ & $35(4.2)$ & Nearly the same \\
\hline Infectious pathology & $36(3.9)$ & $26(3.1)$ & Increased \\
\hline Repeated surgery for postop complications & $17(1.8)$ & $22(2.7)$ & Nearly the same \\
\hline Spontaneous subdural hematoma & $19(2)$ & $34(2.2)$ & Nearly the same \\
\hline Skull base tumors & $44(4.7)$ & $26(3.1)$ & Nearly the same \\
\hline Miscellaneous & $36(3.9)$ & & \\
\hline
\end{tabular}

tients (961 males and 808 females) with a mean age of 37.7 years were evaluated in the study. Surgery was performed in 933 patients during the pre-COVID-19 period at an average of 337.2 patients per month. Surgery was performed in 829 patients during the COVID-19 period at an average of 184.2 per month. During the COVID-19 phase, our department operated at $54.6 \%$ of our pre-COVID-19 capacity (a drop of $45.4 \%$ cases).

The mean ages of surgically treated patients in the pre-COVID-19 and COVID-19 groups were 38.8 and 36.7 years, respectively, with no significant difference. Men constituted $55.4 \%(n=517)$ of the surgically treated patients during the pre-COVID-19 period, compared with $53.6 \%(n=444)$ during the COVID-19 period, with no significant difference.

\section{Types of Surgery}

Table 1 depicts the number of patients who underwent surgery during both periods of time under different diagnostic categories. The percentage of patients treated for a ruptured aneurysm or an arteriovenous malformation (AVM) rose nearly 1.7 times during the COVID-19 period $(18 \%)$ compared with the percentage during the pre-COVID-19 period (10.6\%). Similarly, there was an increased percentage of surgeries performed for hydrocephalus $(31.7 \%$ vs $23.7 \%)$, stroke (6.4\% vs $3.9 \%)$, and cerebellar tumors (4.0\% vs $2.3 \%$ ) during the COVID-19 period, in comparison with percentages during the preCOVID-19 period.

On the other hand, there were reductions in the percentages of patients who were surgically treated for glioma (13\% vs $16.9 \%)$, spinal disorders (4.2\% vs $10.5 \%)$, meningioma $(3.3 \%$ vs $4.2 \%)$, sellar-suprasellar tumors $(2.5 \%$ vs $5.6 \%)$, and cerebral metastasis $(1.7 \%$ vs $6.1 \%)$ during the COVID-19 period, in comparison with percentages during the pre-COVID-19 period.

\section{COVID-19-Positive Cases}

During the COVID-19 period, when a total of 836 nontrauma-related COVID-19 asymptomatic patients were admitted for surgery, 4 patients were found to be positive for the disease on the first COVID-19 testing in the emergency wing area, and an additional 5 patients were found to be positive on the second test, after the first test had indicated no COVID-19 (Table 2).

In contrast to both the local community prevalence of $0.13 \%$ (1315.2 per million in the four states from which most of our patients are referred) and our national cumulative prevalence of $0.2 \%$ (1991.8 per million), the period prevalence of COVID-19 infection among neurosurgery inpatients was $0.48 \%$ (3.7 times more than the community prevalence) with single testing and $1 \%$ with double testing. ${ }^{10,11}$

Two patients - one with a craniopharyngioma (case 1) and another with a meningioma (case 2)-were admitted to the preoperative ward after the first RT-PCR test to detect SARS-CoV-2 yielded negative findings. On the 11th and 12th days after the first test, respectively, their second tests before the planned surgery indicated the presence of COVID-19 simultaneously. Immediately, the preoperative ward was contained, and all close contacts were tested. The mother of the 3-year-old child with a craniopharyngioma (case 1) was also COVID-19 positive. Both the patients and this mother were transferred to the COVID-19 center. Two residents and one faculty member, who had come in contact with these 2 patients, were quarantined. The child was discharged after recovery from COVID-19, in accordance with a preference to undergo surgery at a later time. The patient with a meningioma underwent surgery after 1 month, following two consecutive negative COVID-19 results.

In a case of insular glioma (case 3), the patient's condition deteriorated while he was in the preoperative ward 
TABLE 2. Profiles of COVID-19-positive patients

\begin{tabular}{|c|c|c|c|c|c|c|c|}
\hline $\begin{array}{c}\text { Case } \\
\text { No. }\end{array}$ & $\begin{array}{l}\text { Age } \\
\text { (yrs)/ } \\
\text { Sex }\end{array}$ & Diagnosis & $\begin{array}{c}\text { First COVID-19 } \\
\text { Test at } \\
\text { Admission }\end{array}$ & $\begin{array}{l}\text { Second } \\
\text { COVID-19 } \\
\text { Test }\end{array}$ & $\begin{array}{l}\text { Neurosurgery Area } \\
\text { Where Patient } \\
\text { Tested Positive }\end{array}$ & $\begin{array}{c}\text { No. of Staff } \\
\text { Quarantined } \\
\text { Due to Contact* }\end{array}$ & Final Status \\
\hline 1 & $3 / \mathrm{M} \dagger$ & Craniopharyngioma & Negative & Positive & Preop ward & 1 & Stable, surgery rescheduled \\
\hline 2 & $50 / F$ & Parasagittal meningioma & Negative & Positive & Preop ward & 2 & $\begin{array}{l}\text { Underwent surgery after } 1 \text { month } \\
\text { following } 2 \text { negative tests }\end{array}$ \\
\hline 3 & $70 / \mathrm{M}$ & Insular glioma & Negative & Positive & ICU & 7 & Stable, surgery rescheduled \\
\hline 4 & $45 / M$ & Spinal intradural mass & Negative & Positive & Preop ward & 1 & Stable, surgery rescheduled \\
\hline 5 & $18 / \mathrm{M}$ & $\mathrm{ACoA}$ aneurysm in poor grade & Negative & Positive & Emergency wing & 0 & Plan for surgery once stable \\
\hline 6 & $65 / \mathrm{M}$ & Corpus callosal glioma & Positive & - & Emergency wing & 0 & Stable, surgery rescheduled \\
\hline 7 & $39 / \mathrm{M}$ & Dural arteriovenous fistula & Positive & - & Emergency wing & 0 & Stable, surgery rescheduled \\
\hline 8 & $74 / F$ & $\begin{array}{l}\text { Treated vestibular schwanno- } \\
\text { ma, programmable VP shunt, } \\
\text { diabetes, \& renal failure }\end{array}$ & Positive & - & Emergency wing & 0 & Died \\
\hline 9 & $38 / \mathrm{M}$ & $\begin{array}{l}\text { Tubercular meningitis w/ } \\
\text { hydrocephalus }\end{array}$ & Positive & - & Emergency wing & 0 & $\begin{array}{l}\text { Underwent shunt surgery in } \\
\text { COVID-19 center }\end{array}$ \\
\hline
\end{tabular}

$-=$ not applicable $; \mathrm{ACOA}=$ anterior communicating artery.

* None had COVID-19 symptoms in 2 weeks.

$\dagger$ One family member was determined to have COVID-19 on screening of contacts.

after the first RT-PCR test for SARS-CoV-2 proved negative. The man was transferred to the neurosurgery intensive care unit (ICU) and surgery was planned; the second RT-PCR test indicated the presence of COVID-19. The ICU was immediately contained, and the patient was transferred to the COVID-19 center. All patients and staff in the ICU who had come in close contact with the patient tested negative for the disease. However, seven members of the nursing staff who had attended that patient were quarantined. The patient improved and was later discharged from the COVID-19 center, as he preferred to reschedule the surgery.

A patient with a spinal intradural mass (case 4) was found to have positive findings on the second RT-PCR test performed on the 5th admission day in the preoperative ward. Immediately, the area was contained, and one nursing staff member who came in close contact with the patient was quarantined. All other patients in the ward were tested and found to be negative for the disease. Once the patient had recovered from COVID-19, his surgery was rescheduled, and he was discharged after 10 days.

A fifth patient (case 5) presented with subarachnoid hemorrhage in poor clinical grade. His first test to detect the presence of COVID-19 was negative. The patient was stabilized in the emergency wing area and he underwent CT angiography, which revealed an anterior communicating artery aneurysm. The patient's second test indicated that he had COVID-19. He was transferred to the COVID-19 center, and surgical clipping was planned once the patient's general condition improved.

Four patients (cases 6-9) were found to be COVID-19 positive at the first test and were transferred to the COVID-19 center. In cases 6 (corpus callosal glioma) and 7 (dural arteriovenous fistula), the patients were clinically stable after their recoveries from COVID-19. Their surgeries were rescheduled and the patients were discharged.
The third patient (case 8) had previously been treated for vestibular schwannoma, had received a programmable ventriculoperitoneal (VP) shunt, and had both diabetes and renal failure. Because she presented with hydrocephalus, the shunt pressure setting was adjusted. Following the detection of COVID-19, this patient was transferred to the COVID-19 center, where her general condition continued to deteriorate and she died due to multiorgan dysfunction. The fourth patient (case 9) had tubercular meningitis with hydrocephalus. Since he had COVID-19, he underwent emergency VP shunt surgery in a dedicated operating room in the COVID-19 center, where all attending used PPE, an N95 respirator, and a face shield. The patient improved and was subsequently discharged after testing negative for COVID-19.

All of these patients became COVID-19 positive within 12 days after admission, less than the incubation period, which ranges up to 14 days. While the first 2 patients (cases 1 and 2) tested positive at the same time, none of the patients who shared the area with any COVID-19-positive patients later proved positive for the disease. Additionally, none of the staff caring for these 9 patients developed any symptoms of COVID-19 for up to 2 weeks.

An additional 5 patients tested positive at the second test, following a negative first COVID-19 test. Considering the R0 for COVID-19 in India to be 1.19 and the mean incubation period to be 7 days, and that 8 people in the operating room were exposed to aerosols from each of these 5 patients, it is possible that we may have prevented COVID-19 disease in approximately 67.4 people over a 1-month period, which otherwise might have occurred if surgery had been performed without repeated testing. ${ }^{11,12}$

\section{Discussion}

The worldwide spread of SARS-CoV-2 has put tremen- 
dous pressure on healthcare resources (both human and infrastructure) in all possible ways. A significant portion of healthcare facilities have been selected for the treatment of COVID-19 patients. Some countries have even run out of medical resources in caring for these patients. Many centers have shut down their outpatient departments and restricted elective admissions to preserve their healthcare resources. Since the disease is spread by direct contact and through aerosols, a reduction in the number of cases aims to prevent unnecessary exposure for both patient attendants and healthcare workers, thereby reserving medical professionals for treating COVID-19 patients.

A new strategy for patient screening, admission, and surgical management takes into account the safety of patients, caregivers, and healthcare workers. The hospital administration, in coordination with all subspecialties, is continually formulating criteria for elective and emergency surgeries to prepare and preserve healthcare resources. ${ }^{3,4}$

Neurosurgical practice has also been affected globally due to this pandemic. ${ }^{13-16}$ There is a need for all neurosurgery patients to be tested for COVID-19. Once determined to be COVID-19 positive, these patients should be segregated and treated in a separate zone, away from nonCOVID-19 patients.

In our institution, the number of patients surgically treated during the COVID-19 period dropped from 11.24 neurosurgical procedures per day to 6.03 per day. There have been reports of prioritizing surgical interventions depending on the level of emergency.,17 Delaying surgery can carry the risk of clinical worsening, and objectively categorizing each neurosurgical patient into an emergency or elective is not always possible.

Considering the evolving nature of a pandemic, a specific period of nonoperative intervention without the risk of further deterioration cannot be ensured and may also have legal implications. In India, although the lockdown was implemented with a phased easing of restrictions, the number of positive cases is still increasing. Being the second-most populous country in the world, even the small percentage of neurosurgical patients comprises considerable numbers. Thus, emergency services continue to be provided as before, and elective surgeries are prioritized. To attend cross-referrals from various centers, we have started telephone consultations three times per week.

Additionally, patients discharged from our department during this pandemic are also followed up through the use of this telephone consultation service, including neuropsychological support. ${ }^{18,19}$ This has reduced the follow-up caseload tremendously. The lockdown and the panic associated with COVID-19 have changed the types of patients visiting our institution. Surgery for benign lesions, spinal disorders, and functional diseases has decreased significantly, probably due to economic constraints. ${ }^{20,21}$ Surgery for sellar-suprasellar tumors has also declined, except for patients with worsening visual complaints, due to concerns of viral load in the sinus mucosa and aerosol generation during surgery. ${ }^{22,23}$ The percentages of patients with subarachnoid hemorrhage, stroke, hydrocephalus, and cerebellar tumors have increased during this pandemic, as most of these patients have an acute presentation. Some of these illnesses, including subarachnoid hemorrhage and stroke, are also correlated with a sedentary lifestyle, dietary factors, and the high stress levels expected in the pandemic. ${ }^{24-26}$

It is pertinent to mention that patients who required urgent intervention received surgery following one negative test for COVID-19 at admission. Stable patients who were scheduled for surgery at a later date were kept in the preoperative area, and a second COVID-19 test was performed within 48 hours before surgery. By following this protocol, we were able to detect an additional 5 patients after a first negative report for SARS-CoV-2. This repeated testing helped us further isolate all contacts among other patients and health workers to prevent additional COVID-19 transmission.

The inpatient infection rate detected in non-traumarelated neurosurgery patients in our study is significantly higher than both our local community prevalence and our country prevalence of 1991.8 per million population..$^{11}$ It is possible that many patients have come from neighboring states and have visited several hospitals seeking medical advice. The many travels and potential points of contact thus may be the possible cause of this significantly higher COVID-19 positivity rate among our neurosurgery patients.

The second test of admitted patients helped us to restrict the spread of infection and thereby save our healthcare resources. None of the other patients subsequently tested positive for the disease. So, after a second negative test, patients can be considered relatively safe with very little chance of COVID-19 spread during the postoperative period.

As this virus is spread through aerosols, use of negative pressure suction rooms is reported for performing surgical procedures. ${ }^{3}$ In developing countries, these facilities may not be available at all surgical centers. So, repeated testing may be a practical containment measure among inpatients. A single negative test for emergency procedures and double negative tests for elective cases ensure the safety of surgeons, assistants, and technicians in the operating room. In the recovery wards, all nursing staff and attending doctors should take protective measures, including masks, face shields, and gloves. The use of a face shield has been made mandatory for airway suctioning in all areas.

It is not possible to maintain a zero-infection rate among healthcare workers and admitted patients, given the highly infectious nature of this virus and the long incubation period. ${ }^{3}$ A single entry zone into the hospital ensures proper screening and evaluation for the need for admission, further facilitating sample collection and contact tracing.

The reason why a second test can be positive after a negative first test could be a false-negative first test, insufficient viral load in the early phase of the incubation period, or hospital-acquired COVID-19 infection. ${ }^{27}$ The patients in our study became disease positive within the incubation period of up to 2 weeks from admission, thereby indicating community-acquired COVID-19. ${ }^{10}$ To reduce an in-hospital outbreak, educating patients' caretakers about precautionary measures is essential. Their mobility has to be restricted, and proper use of face masks, hand sanitization, and maintenance of social distancing should be monitored strictly. Another option is to convert each 
general ward into different cubicles and restrict the number of caretakers to prevent reinfection among neurosurgical inpatients.

All healthcare workers also need to be educated about COVID-19 spread patterns and methods of prevention. The knowledge of COVID-19 infection control must be translated into practice. ${ }^{28,29}$ The limitations of our study include the noncontrolled design, short period of assessment, and single-center evaluation, which can limit its generalizability. We have not included neurotrauma cases, as many of these patients would have received emergency care in other centers before reaching us. ${ }^{17,30}$ However, considering the absence of any good quality evidence on this topic, our study is highly pertinent.

\section{Conclusions}

The restrictions imposed by COVID-19 have resulted in a significant change in the pattern of neurosurgical procedures, with acute cases dominating over chronic benign disorders. India has yet to reach the peak of the pandemic. The period prevalence of COVID-19 with single testing in asymptomatic neurosurgical inpatients is 3.7 times higher than in the community. Double COVID-19 testing has incremental value, disclosing COVID-19 overall in 1 of 100 inpatients, and is useful in averting possible infection spread through neurosurgical services.

\section{References}

1. Government of India. COVID 19 state wise status. About COVID prevalence in community. Accessed October 12, 2020. https://www.mygov.in/corona-data/covid19-statewisestatus

2. Germanò A, Raffa G, Angileri FF, et al. Coronavirus disease 2019 (COVID-19) and neurosurgery: literature and neurosurgical societies recommendations update. World Neurosurg. 2020;139:e812-e817.

3. Flemming S, Hankir M, Ernestus RI, et al. Surgery in times of COVID-19-recommendations for hospital and patient management. Langenbecks Arch Surg. 2020;405(3):359-364.

4. Jean WC, Ironside NT, Sack KD, et al. The impact of COVID-19 on neurosurgeons and the strategy for triaging non-emergent operations: a global neurosurgery study. Acta Neurochir (Wien). 2020;162(6):1229-1240.

5. Bartoszko JJ, Farooqi MAM, Alhazzani W, Loeb M. Medical masks vs N95 respirators for preventing COVID-19 in healthcare workers: a systematic review and meta-analysis of randomized trials. Influenza Other Respir Viruses. 2020; 14(4):365-373.

6. van Kasteren PB, van der Veer B, van den Brink S, et al. Comparison of seven commercial RT-PCR diagnostic kits for COVID-19. J Clin Virol. 2020;128:104412.

7. Ai T, Yang Z, Hou H, et al. Correlation of chest CT and RTPCR testing for coronavirus disease 2019 (COVID-19) in China: a report of 1014 Cases. Radiology. 2020;296(2):E32E40.

8. Cao H, Ruan L, Liu J, Liao W. The clinical characteristic of eight patients of COVID-19 with positive RT-PCR test after discharge. J Med Virol. 2020;92(10):2159-2164.

9. Lepelletier D, Grandbastien B, Romano-Bertrand S, et al. What face mask for what use in the context of COVID-19 pandemic? The French guidelines. J Hosp Infect. 2020; 105(3):414-418.

10. India's COVID-19 infection rate has increased: what this means. About reproduction rate of COVID-19. Times Now
Digital. July 9, 2020. Accessed October 12, 2020. https: //www.timesnownews.com/india/article/india-s-covid19-transmission-rate-has-increased-what-this-means/618799

11. Backer JA, Klinkenberg D, Wallinga J. Incubation period of 2019 novel coronavirus (2019-nCoV) infections among travellers from Wuhan, China, 20-28 January 2020. Euro Surveill. 2020;25(5):2000062.

12. Unique Identification Authority of India. About population of states. Accessed October 12, 2020. https://uidai.gov.in/ images/state-wise-aadhaar-saturation.pdf

13. Cenzato M, DiMeco F, Fontanella M, et al. Editorial. Neurosurgery in the storm of COVID-19: suggestions from the Lombardy region, Italy (ex malo bonum). J Neurosurg. 2020; 133(1):33-34.

14. Tan YT, Wang JW, Zhao K, et al. Preliminary recommendations for surgical practice of neurosurgery department in the central epidemic area of 2019 coronavirus infection. Curr Med Sci. 2020;40(2):281-284.

15. Gupta P, Muthukumar N, Rajshekhar V, et al. Neurosurgery and neurology practices during the novel COVID-19 pandemic: a consensus statement from India. Neurol India. 2020; 68(2):246-254.

16. Lee ZD, Chyi Yeu DL, Ang BT, et al. Editorial. COVID-19 and its impact on neurosurgery: our early experience in Singapore. J Neurosurg. 2020;133(1):24-25.

17. De Simone B, Chouillard E, Di Saverio S, et al. Emergency surgery during the COVID-19 pandemic: what you need to know for practice. Ann R Coll Surg Engl. 2020;102(5): $323-332$

18. Dhandapani M, Gupta S, Mohanty M, et al. Prevalence and trends in the neuropsychological burden of patients having intracranial tumors with respect to neurosurgical intervention. Ann Neurosci. 2017;24(2):105-110.

19. Liu N, Huang R, Baldacchino T, et al. Telehealth for noncritical patients with chronic diseases during the pandemic. J Med Internet Res. 2020;22(8):e19493.

20. Chowdhury R, Luhar S, Khan N, et al. Long-term strategies to control COVID-19 in low and middle-income countries: an options overview of community-based, non-pharmacological interventions. Eur J Epidemiol. 2020;35(8):743-748.

21. Dhandapani SS, Manju D, Mahapatra AK. The economic divide in outcome following severe head injury. Asian $\mathrm{J} \mathrm{Neu-}$ rosurg. 2012;7(1):17-20.

22. Patil NR, Dhandapani S, Sahoo SK, et al. Differential independent impact of the intraoperative use of navigation and angled endoscopes on the surgical outcome of endonasal endoscopy for pituitary tumors: a prospective study. Neurosurg Rev. Published online October 22, 2020. doi:10.1007/s10143020-01416-x

23. Workman AD, Welling DB, Carter BS, et al. Endonasal instrumentation and aerosolization risk in the era of COVID-19: simulation, literature review, and proposed mitigation strategies. Int Forum Allergy Rhinol. 2020;10(7): 798-805.

24. Dhandapani S, Kapoor A, Gaudihalli S, et al. Study of trends in anthropometric nutritional indices and the impact of adiposity among patients of subarachnoid hemorrhage. Neurol India. 2015;63(4):531-536.

25. Dhandapani S, Singh A, Singla N, et al. Has outcome of subarachnoid hemorrhage changed with improvements in neurosurgical services? Study of 2000 patients over 2 decades from India. Stroke. 2018;49(12):2890-2895.

26. Sarfo FS, Mensah NO, Opoku FA, et al. COVID-19 and stroke: experience in a Ghanaian healthcare system. J Neurol Sci. 2020;416:117044.

27. Li Y, Yao L, Li J, et al. Stability issues of RT-PCR testing of SARS-CoV-2 for hospitalized patients clinically diagnosed with COVID-19. J Med Virol. 2020;92(7):903-908.

28. Zhang M, Zhou M, Tang F, et al. Knowledge, attitude, and 
practice regarding COVID-19 among healthcare workers in Henan, China. J Hosp Infect. 2020;105(2):183-187.

29. Issac A, Stephen S, Jacob J, et al. The Pandemic League of COVID-19: Korea Versus the United States, With Lessons for the Entire World. J Prev Med Public Health. 2020;53(4): 228-232.

30. Mukherjee KK, Dhandapani S, Sarda AC, et al. Prospective comparison of simple suturing and elevation debridement in compound depressed fractures with no significant mass effect. Acta Neurochir (Wien). 2015;157(2):305-309.

\section{Disclosures}

The authors report no conflict of interest concerning the materials or methods used in this study or the findings specified in this paper.

\section{Author Contributions}

Conception and design: Dhandapani. Acquisition of data: Dhandapani, Sahoo. Analysis and interpretation of data: Dhandapani. Drafting the article: Dhandapani, Sahoo. Critically revising the article: Dhandapani. Reviewed submitted version of manuscript: all authors. Approved the final version of the manuscript on behalf of all authors: Dhandapani. Statistical analysis: Dhandapani. Administrative/technical/material support: Dhandapani, Singh, Gendle, Karthigeyan, Salunke, Aggarwal, N Singla, R Singla, Tripathi, Chhabra, Mohindra, Tewari, Mohanty, Bhagat, Chakrabarti, Gupta. Study supervision: Dhandapani, Gupta.

\section{Correspondence}

Sivashanmugam Dhandapani: Postgraduate Institute of Medical Education and Research, Chandigarh, India. ssdhandapani. neurosurg@gmail.com. 